\title{
Baicalein reverses hypoxia-induced 5-FU resistance in gastric cancer AGS cells through suppression of glycolysis and the PTEN/Akt/HIF-1 $\alpha$ signaling pathway
}

\author{
FENGLIN CHEN $^{1 *}$, MINGKAI ZHUANG $^{1 *}$, CANMEI ZHONG ${ }^{2}, \mathrm{JUN} \mathrm{PENG}^{3}, \mathrm{XIAOZHONG} \mathrm{WANG}^{1}$, \\ JIANYING LI $^{1}$, ZHIXIN CHEN ${ }^{1}$ and YUEHONG HUANG ${ }^{1}$ \\ ${ }^{1}$ Department of Gastroenterology, Union Hospital of Fujian Medical University, Fuzhou, Fujian 350001; \\ ${ }^{2}$ College of Union Clinical Medicine, Fujian Medical University, Fuzhou, Fujian 350001; \\ ${ }^{3}$ Academy of Integrative Medicine Biomedical Research Center, Fujian University of \\ Traditional Chinese Medicine, Fuzhou, Fujian 350001, P.R. China
}

Received July 2, 2014; Accepted September 25, 2014

DOI: $10.3892 /$ or.2014.3550

\begin{abstract}
Cancer cells can survive under hypoxia by metabolic reprogramming to achieve a high level of glycolysis, which contributes to the development of chemoresistance. Therefore, inhibition of glycolysis would be a novel strategy for overcoming hypoxia-induced drug resistance. Baicalein, a flavonoid derived from the root of Scutellaria baicalensis, has been reported to exert strong antitumor activity toward various types of cancer. In the present study, we evaluated the effect of baicalein on hypoxia-induced 5-fluorouracil (5-FU) resistance in gastric cancer AGS cells and investigated the possible molecular mechanisms. We found that baicalein increased the sensitivity of AGS cells to 5-FU treatment under hypoxia. In addition, the hypoxia-enhanced glycolytic flux and expression of several critical glycolysis-associated enzymes (HK2, LDH-A and PDK1) in the AGS cells were suppressed by baicalein. Furthermore, baicalein inhibited hypoxia-induced Akt phosphorylation by promoting PTEN accumulation, thereby attenuating hypoxia-inducible factor- $1 \alpha$ (HIF-1 $\alpha)$ expression in AGS cells. These results together suggest that inhibition of glycolysis via regulation of the PTEN/Akt/HIF-1 $\alpha$ signaling pathway may be one of the mechanisms whereby baicalein reverses 5-FU resistance in cancer cells under hypoxia.
\end{abstract}

\section{Introduction}

Gastric cancer (GC) is one of the leading causes of cancerrelated mortality, accounting for more than 700,000 deaths

Correspondence to: Dr Xiaozhong Wang, Department of Gastroenterology, Union Hospital of Fujian Medical University, 29 Xinquan Road, Fuzhou, Fujian 350001, P.R. China

E-mail: drwangxz@pub6.fz.fj.cn

*Contributed equally

Key words: baicalein, hypoxia, 5-flurouracil, chemoresistance, glycolysis, gastric carcinoma worldwide annually (1). Although great efforts have been invested to treat GC in the clinic, the overall 5-year survival rate of GC patients is only $20-25 \%$, with a median survival time of $\sim 24$ months (2). Resistance to chemotherapy is one of the important factors responsible for the poor prognosis of GC. 5-Fluorouracil (5-FU) is an important chemotherapeutic drug used for the systemic treatment of GC. 5-FU exerts its anticancer effects through inhibition of thymidylate synthase and incorporation of its metabolites into DNA or RNA. Multiple clinical trials have demonstrated that single or combination therapy based on 5-FU confers survival benefits for GC patients $(3,4)$. However, there are a number of GC patients resistant to 5-FU treatment, which is a main reason for the failure of clinical GC chemotherapy.

The fast growth of cancer cells and the rapid expansion of the tumor mass usually outpace new vascular generation, resulting in an insufficient blood supply to certain areas of the tumor tissues. Therefore, a hypoxic microenvironment is present in most solid tumors. Growing evidence shows that hypoxia has a negative impact on chemotherapy (5). Hypoxiainducible factor- $1 \alpha$ (HIF-1 $\alpha$ ) plays an essential role in the adaptive response of tumor cells to hypoxia and is associated with the development of hypoxia-induced resistance to chemotherapeutic drugs. Recently, HIF-1 $\alpha$ has been shown to be overexpressed in a variety of tumors including GC and to enhance 5-FU drug resistance resulting in the poor response of GC patients to 5-FU-based chemotherapy $(6,7)$. HIF-1 $\alpha$ regulates more than 60 target genes involved in cell proliferation, apoptosis, angiogenesis and glycolytic metabolism (8-10). Of these phenotypes, the activation of the glycolytic pathway is a necessary one that enables tumor cells to survive under hypoxia. A previous study demonstrated that a high rate of glycolysis is one of the major causative factors for hypoxiainduced drug resistance in cancer cells (11). Therefore, inhibition of HIF-1 $\alpha$ and glycolysis in cancer cells appears to be a potential strategy for the reversal of hypoxia-induced resistance to chemotherapy.

Hypoxia initiates intracellular signaling pathways, leading to the activation of HIF-1 $\alpha$ in tumor cells. Akt is one of the 


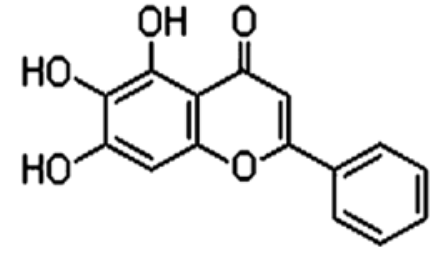

Figure 1. Chemical structure of baicalein.

important regulatory pathways controlling HIF-1 $\alpha$ protein levels, which therefore may be a promising therapeutic target for overcoming HIF-1 $\alpha$-mediated apoptotic resistance in tumor cells under hypoxia (12-16). PTEN is a tumor-suppressor functioning as an antagonist of the Akt signaling pathway. Loss of PTEN has been found to augment HIF-1 $\alpha$-mediated gene expression, and restoration of PTEN can inhibit the expression of HIF-1 $\alpha$ (17).

Baicalein (Fig. 1), a flavonoid derived from the root of Scutellaria baicalensis, has been revealed to possess a wide variety of biological activities, such as antitumor, anti-microbe, anti-inflammatory and anti-ischemic properties (18-21). Since it is a promising anticancer drug, it is important to evaluate the beneficial effect of baicalein as a single treatment or when administered in combination with other conventional anticancer drugs. In a previous study, Taniguchi et al found that baicalein overcomes tumor necrosis factor-related apoptosis-inducing ligand resistance in cancer cells through DR5 upregulation mediated by ROS induction and CHOP/GADD153 activation (22). However, the effects of baicalein on hypoxia-induced resistance to chemotherapeutic drugs and the related mechanisms in GC cells have not yet been determined.

In the present study, using a human GC cell line AGS, we found that baicalein decreased the glycolytic rate through reducing the expression of hexokinase-2 (HK2), lactate dehydrogenase A (LDHA) and pyruvate dehydrogenase kinase-1 (PDK1) under a hypoxic condition, leading to the reversal of hypoxia-induced resistance to 5-FU in AGS cells. In addition, baicalein treatment suppressed HIF-1 $\alpha$ expression and this process was at least partly due to the upregulation of PTEN and downregulation of Akt phosphorylation. Collectively, it is suggested that inhibition of glycolysis of cancer cells via modulation of the PTEN/Akt/HIF-1 $\alpha$ signaling pathway could be one of the mechanisms whereby baicalein overcomes the hypoxia-induced resistance of GC cells to chemotherapeutic drugs.

\section{Materials and methods}

Reagents and antibodies. Baicalein was purchased from Enzo (Farmingdale, NY, USA); 5-FU was obtained from Sigma; primary antibodies for HIF-1 $\alpha$, p-Akt, Akt, PTEN, HK2, PDK1, LDHA, $\beta$-actin and secondary antibodies, HRP-conjugated goat anti-rabbit IgG were purchased from Cell Signaling Technology (Beverly, MA, USA).

Cell culture. The human GC cell line AGS was purchased from KeyGen (Nanjing, Jiangsu, China). Cells were cultured with RPMI-1640 medium (Life Technologies, Grand Island, NY,
USA) supplemented with $10 \%$ fetal bovine serum, $100 \mathrm{U} / \mathrm{ml}$ penicillin and $100 \mu \mathrm{g} / \mathrm{ml}$ streptomycin in a humidified incubator at $37^{\circ} \mathrm{C}$ under $5 \% \mathrm{CO}_{2}$ in air. For hypoxia experiments $\left(0.2 \% \mathrm{O}_{2}\right)$, cells were incubated in an airtight chamber flushed with a gas mixture containing $10 \% \mathrm{CO}_{2}, 10 \% \mathrm{H}_{2}$ and $80 \% \mathrm{~N}_{2}$.

Drug preparation. Baicalein and 5-FU were dissolved in dimethyl sulfoxide (DMSO) to prepare stock solutions. The working concentrations of baicalein and 5-FU were made by diluting the stock solutions in the complete culture medium. The final DMSO concentration of solution used in all experiments did not exceed $0.45 \%$.

Cytotoxicity assay. The cytotoxicity of baicalein on AGS cells under hypoxia was examined by 3-(4,5-dimethylthiazol2-yl)-2,5-diphenyltetrazolium bromide (MTT) assay. In brief, AGS cells were seeded into 96 -well plates at a density of $4 \times 10^{3}$ cells/well in $0.1 \mathrm{ml}$ medium overnight prior to a $24-$ or 48-h treatment with increasing concentrations of baicalein $(0$, $10,20,40,60$ and $80 \mu \mathrm{M})$ under hypoxia. After treatment, MTT solution was added into each well and the plates were incubated for an additional $4 \mathrm{~h}$, allowing viable cells to reduce the yellow MTT into purple-blue formazan crystals. Finally, DMSO was used to dissolve the formazan crystals. The absorbance was measured at $570 \mathrm{~nm}$ by a microplate reader (ELx800; BioTek, Winooski, VT, USA). The cell viability was calculated according to the formula: $\mathrm{OD}_{\mathrm{drug}} / \mathrm{OD}_{\text {control }} \times 100 \%$.

Analysis of drug resistance and reversal effect. The effect of hypoxia on sensitivity to 5-FU and the ability of baicalein to reverse hypoxia-induced 5-FU resistance in AGS cells were evaluated using the MTT assay. AGS cells in log-phase were harvested and seeded into 96 -well plates $\left(4 \times 10^{3}\right.$ cells/well $)$ overnight. Increasing concentrations of 5-FU $(0,125,250,500$, 1,000 and $2,000 \mu \mathrm{M}$ ) or combinations of 5-FU with baicalein $(10,20$ and $40 \mu \mathrm{M})$ were added and further incubated for $48 \mathrm{~h}$ under normoxic or hypoxic conditions. The cell viability was determined using the MTT assay as mentioned before. The concentration resulting in $50 \%$ inhibition of cell viability $\left(\mathrm{IC}_{50}\right)$ of 5-FU was calculated by GraphPad Prism 5 software. In addition, the resistant index (RI) and reversal fold (RF) were calculated as follow:

$$
\begin{gathered}
\mathrm{RI}=\mathrm{IC}_{50} \text { of } 5-\mathrm{FU}_{\text {under hypoxia }} / \mathrm{IC}_{50} \text { of } 5-\mathrm{FU}_{\text {under normoxia }} \\
\mathrm{RF}=\mathrm{IC}_{50} \text { of } 5-\mathrm{FU}_{\text {under hypoxia }} / \mathrm{IC}_{50} \text { of } 5-\mathrm{FU}+\text { baicalein }_{\text {under hypoxia }}
\end{gathered}
$$

Measurement of the metabolic rate of glucose and lactate. The glucose uptake and lactate production assay was performed as previously described with some modifications (23). In brief, cells were seeded in $25 \mathrm{~cm}^{2}$ flasks ( $8 \times 10^{5}$ cells/flask) and treated with different concentrations of baicalein $(0,10$, 20 and $40 \mu \mathrm{M})$ under normoxic or hypoxic conditions for $24 \mathrm{~h}$. Then the culture medium was collected. The concentrations of glucose and lactate in the medium were respectively measured by an Amplex Red Glucose/Glucose Oxidase assay kit (Life Technologies) and a Lactic Acid assay kit (KeyGen, Nanjing, China), according to the manufacturer's instructions. The absorbance at $560 \mathrm{~nm}$ (glucose) or at $530 \mathrm{~nm}$ (lactate) was respectively detected using a microplate reader or a spectrophotometer. Consumption of glucose and production of lactate were calculated from the difference between the 
Table I. Real-time qPCR primers.

Sequence of primers (5' to $3^{\prime}$ )

\begin{tabular}{ll} 
HK2 & Forward: TGCCACCAGACTAAACTAGACG \\
& Reverse: CCCGTGCCCACAATGAGAC \\
LDHA & Forward: ATGGCAACTCTAAAGGATCAGC \\
& Reverse: CCAACCCCAACAACTGTAATCT \\
PDK1 & $\begin{array}{l}\text { Forward: CTGTGATACGGATCAGAAACCG } \\
\text { Reverse: TCCACCAAACAATAAAGAGTGCT }\end{array}$ \\
\multirow{3}{*}{-actin } & Forward: CCTCCATCGTCCACCGCAAAT \\
& Reverse: GCTGTCACCTTCACCGTTCCA
\end{tabular}

HK2, hexokinase-2; LDHA, lactate dehydrogenase A; PDK1, pyruvate dehydrogenase kinase- 1 .

concentrations in the medium at the beginning and at the end of drug treatment.

Real-time quantitative polymerase chain reaction ( $q P C R)$. After AGS cells achieved treatment as mentioned previously, total RNA was extracted from cells using RNAiso reagent (Takara, Dalian, China) following the manufacturer's protocol. cDNA was synthesized from $1 \mu \mathrm{g}$ of total RNA using the PrimeScript $^{\mathrm{TM}}$ RT reagent kit (Takara). Real-time quantitative PCR analysis was performed using DyNAmo ColorFlash SYBR-Green qPCR kit (Thermo Fisher, Waltham, MA, USA) with 7500 Fast Real-Time PCR System (Applied Biosystems, Foster City, CA, USA). The data were analyzed using the $2^{-\Delta \Delta \mathrm{Ct}}$ method. The gene expression levels were normalized to $\beta$-actin and are presented as relative fold change compared to the control. All reactions were independently repeated three times and the average was calculated. The sequences of the primers are shown in Table I.

Western blotting. After AGS cells achieved treatment as mentioned previously, the cells were lysed in RIPA buffer (Pierce, Rockford, IL, USA) with complete protease inhibitors and PhosSTOP Phosphatase Inhibitor (Roche, Mannheim, Germany). Then the lysates were clarified by centrifugation at $4^{\circ} \mathrm{C}$ for $15 \mathrm{~min}$ at $14,000 \mathrm{rpm}$. The concentration of protein in the supernatants was detected using the BCA protein assay kit (Pierce). Then an equal amount of proteins was separated by $10 \%$ SDS-PAGE gels and transferred onto PVDF membranes (Roche). The PVDF membranes were blocked with $3 \%$ bovine serum albumin for $1.5 \mathrm{~h}$ and probed with the indicated primary antibodies overnight at $4^{\circ} \mathrm{C}$. After three time washing with TBST, the membranes were incubated with the appropriate HRP-conjugated secondary antibody for $1 \mathrm{~h}$ at room temperature. The bands were detected using enhanced chemiluminescence (Pierce).

Statistical analysis. All experiments were repeated at least three times. Data are presented as means \pm SD. The differences between groups were analyzed by the Student's t-test or one-way ANOVA using SPSS 19.0 software. A probability value $\mathrm{P}<0.05$ was considered to indicate a statistically significant result.

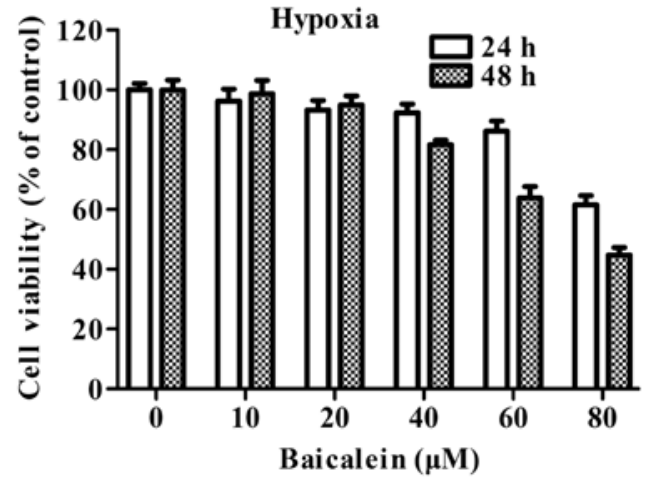

Figure 2. Cytotoxic effect of baicalein on AGS cells under hypoxia. After treatment with the indicated concentrations of baicalein under hypoxia for 24 or $48 \mathrm{~h}$, cell viability was analyzed by the MTT assay. The data were normalized to the absorbance of the control group. Date are represented as means $\pm \mathrm{SD}$ of three independent experiments.

\section{Results}

Cytotoxic effect of baicalein on AGS cells under hypoxic conditions. The cytotoxic effect of baicalein on AGS cells under hypoxia was examined by MTT assay. The result indicated that baicalein inhibited the proliferation of AGS cells in a concentration-dependent manner under hypoxia. According to the dose-effect curve (Fig. 2), 10 and $20 \mu \mathrm{M}$ baicalein were not cytotoxic to AGS cells at both 24 and $48 \mathrm{~h}$ (cell viability $>90 \%$ ), $40 \mu \mathrm{M}$ baicalein had a weak cytotoxic effect on AGS cells at $48 \mathrm{~h}$ (cell viability $>80 \%$ ), while 60 and $80 \mu \mathrm{M}$ baicalein exhibited obvious cytotoxicity on AGS cells at $48 \mathrm{~h}$ (cell viability $<65 \%$ ). Based on the above data, we employed 10 , 20 and $40 \mu \mathrm{M}$ as optimum concentrations of baicalein for the subsequent studies.

Baicalein reverses hypoxia-induced 5-FU resistance in AGS cells. The effect of hypoxia on the sensitivity of AGS cells to 5-FU and the ability of baicalein to reverse hypoxia-induced 5-FU resistance in AGS cell were evaluated using the MTT assay. As shown in Fig. 3A, the cytotoxic effect of 5-FU on AGS cells was significantly weakened under a hypoxic condition. The RI by hypoxia was 2.65 (Table II). These results indicate that a hypoxic condition enhanced the resistance of AGS cells to 5-FU. The addition of baicalein attenuated the increased resistance of the AGS cells to 5-FU under a hypoxic condition; the responsiveness was reduced to the level observed under a normoxic condition and even lower (Fig. 3B). In addition, the $\mathrm{RF}$ of baicalein at 10,20 and $40 \mu \mathrm{M}$ was $3.09,5.60$ and 10.05, respectively (Table II).

An $R F>1$ indicates enhanced drug sensitivity in the presence of a modulator and the greater the RF magnitude, the more significant is the reversal effect.

Baicalein inhibits glucose uptake and lactate production of AGS cells under a hypoxic condition. It is well known that hypoxia enhances the glycolytic flux of cancer cells, which has been demonstrated to be responsible for drug resistance associated with hypoxia. Thus, we tested the effect of baicalein on glycolysis in AGS cells under a hypoxic condition. The glycolytic efficiency of cells are reflected by the rates of glucose 
Table II. Reversal effect of baicalein on hypoxia-induced 5-FU resistance in AGS cells.

\begin{tabular}{|c|c|c|c|c|}
\hline Treatment & $\begin{array}{l}\mathrm{IC}_{50}(\mu \mathrm{M}) \\
\text { (normoxia) }\end{array}$ & $\begin{array}{l}\mathrm{IC}_{50}(\mu \mathrm{M}) \\
\text { (hypoxia) }\end{array}$ & RI & $\mathrm{RF}$ \\
\hline 5-FU & $562.9 \pm 45.2$ & $1,495 \pm 356.5^{\mathrm{a}}$ & 2.65 & - \\
\hline $\begin{array}{c}5-\mathrm{FU}+\text { baicalein } \\
(10 \mu \mathrm{M})\end{array}$ & - & $482.9 \pm 38.5^{\mathrm{b}}$ & - & 3.09 \\
\hline $\begin{array}{c}5-\mathrm{FU}+\text { baicalein } \\
(20 \mu \mathrm{M})\end{array}$ & - & $266.8 \pm 25.1^{\mathrm{b}}$ & - & 5.60 \\
\hline $\begin{array}{c}5-\mathrm{FU}+\text { baicalein } \\
(40 \mu \mathrm{M})\end{array}$ & - & $148.8 \pm 26.8^{b}$ & - & 10.05 \\
\hline
\end{tabular}

AGS cells were treated with various concentrations of 5-FU in the present of $0,10,20$ and $40 \mu \mathrm{M}$ of baicalein for $48 \mathrm{~h}$ under normoxia or hypoxia. $\mathrm{IC}_{50}$ values for 5-FU, the resistance index (RI) and the reversal fold (RF) were calculated. Data are represented as means $\pm \mathrm{SD}$ of three independent experiments. ${ }^{\mathrm{P}}<0.05$ vs. normoxia; ${ }^{b} \mathrm{P}<0.05$ vs. 5-FU alone. 5-FU, 5-fluorouracil.
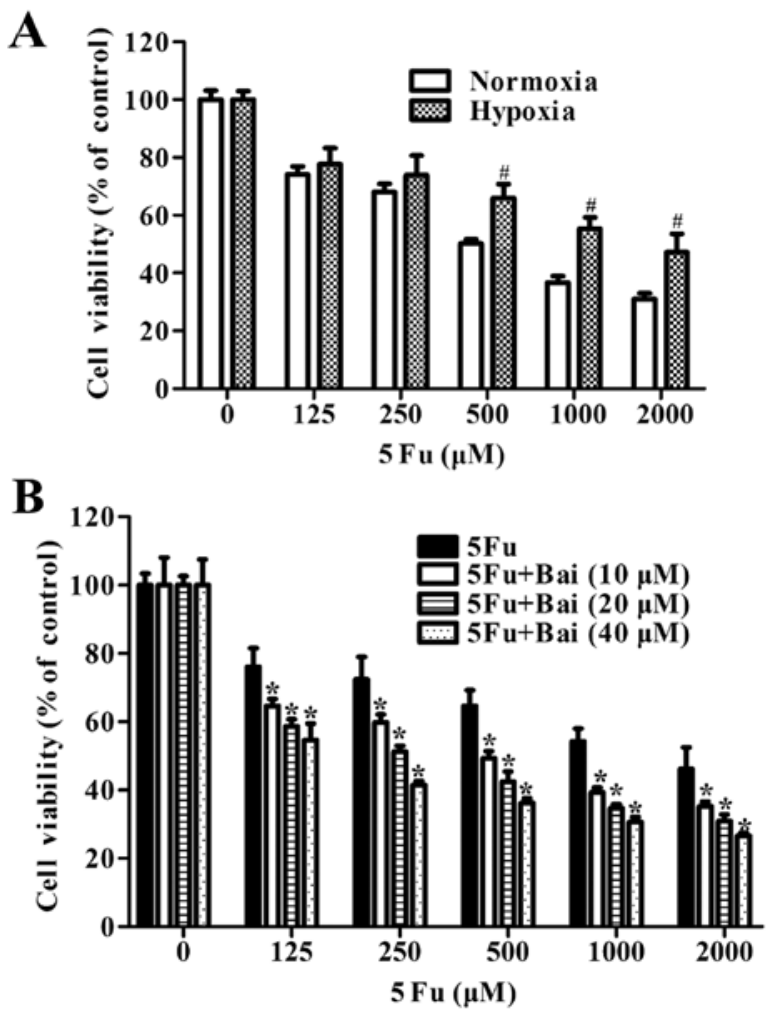

Figure 3. Baicalein reverses hypoxia-induced resistance to 5-FU in AGS cells. (A) AGS cells were treated with the indicated concentration of 5-FU for $48 \mathrm{~h}$ under normoxia or hypoxia. (B) AGS cells were treated with the indicated concentrations of 5-FU in the presence of $0,10,20$ and $40 \mu \mathrm{M}$ baicalein (Bai) for $48 \mathrm{~h}$ under hypoxia. Cell viability after treatment was analyzed by MTT assay. The data were normalized to the absorbance of the control group. Data are represented as means $\pm \mathrm{SD}$ of three independent experiments. ${ }^{\prime \prime} \mathrm{P}<0.05$ compared with the normoxia group treated with the same concentration of 5 -FU; ${ }^{*} \mathrm{P}<0.05$ compared with the 5 -FU alone group treated with the same concentration of 5-FU. 5-FU, 5-fluorouracil.

uptake and lactate production. Our results showed that AGS cells consumed more glucose and generated more lactate for
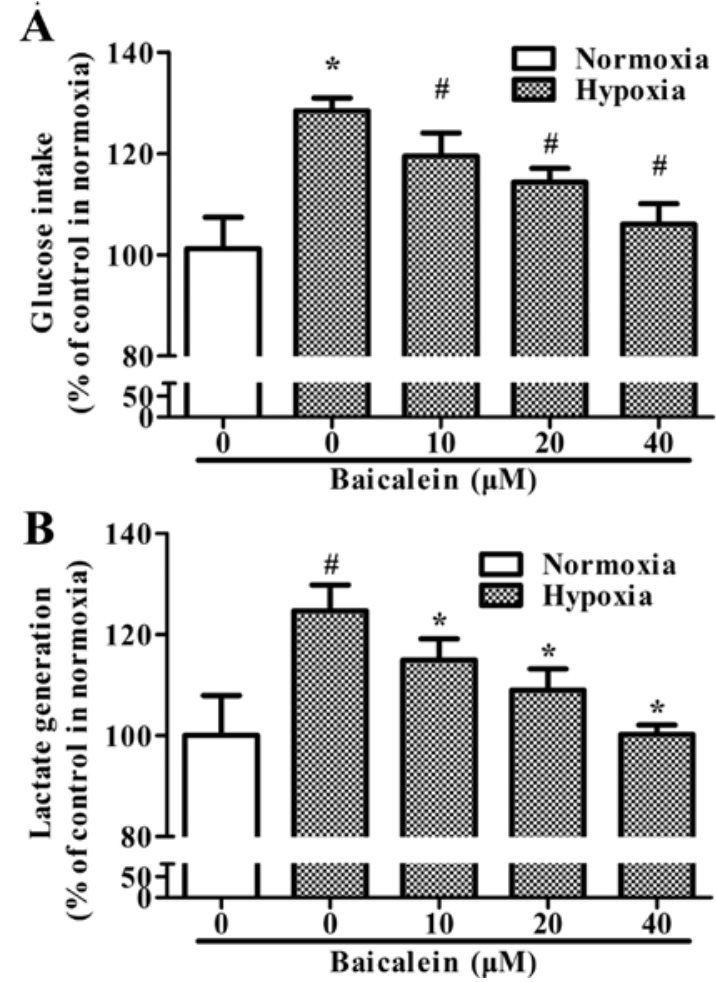

Figure 4. Baicalein inhibits glucose uptake and lactate production of AGS cells under hypoxia. AGS cells were treated with the indicated concentrations of baicalein under normoxia or hypoxia. Glucose uptake (A) and lactate production (B) were determined as described in Materials and methods. Data are represented as means $\pm \mathrm{SD}$ of three independent experiments. The data were normalized to the absorbance of the normoxic group. ${ }^{\text { }} \mathrm{P}<0.05$ compared with the normoxic group; $\mathrm{P}<0.05$ compared with the $5-\mathrm{FU}$ hypoxic group treated without baicalein. 5-FU, 5-fluorouracil.

survival and growth under a hypoxic condition compared with that under a normoxic condition. Nevertheless, the increased glucose uptake and lactate production under hypoxia gradually returned to the levels under normoxia after treatment with baicalein (Fig. 4). These results imply that baicalein retarded the accelerated glycolytic rate induced by hypoxia.

Baicalein inhibits the expression of glycolysis-related enzymes in AGS cells under a hypoxic condition. Next, we investigated the enzymes that may participate in baicaleininduced glycolysis inhibition under a hypoxic condition. Using qPCR and western blotting, we found that baicalein profoundly attenuated the expression of hypoxia-induced glycolysis-related enzymes (HK2, LDHA and PDK1) in a dose-dependent manner (Fig. 5).

Baicalein inhibits hypoxia-dependent HIF-1a expression via regulation of the PTEN/Akt pathway in AGS cells. Since HIF-1 $\alpha$ is the major transcriptional factor activated by hypoxia and activation of HIF-1 $\alpha$ amplifies glycolysis by increasing the synthesis of glycolytic enzymes, we hypothesized that HIF-1 $\alpha$ may be involved in the inhibition of baicalein to glycolysis. As a result, baicalein reduced the protein level of HIF- $1 \alpha$ in a dose-dependent manner under a hypoxic condition.

Considering the importance of the Akt signaling pathway in regulating hypoxic responses of tumor cells, we investigated the effect of baicalein on the phosphorylation of Akt in 
A
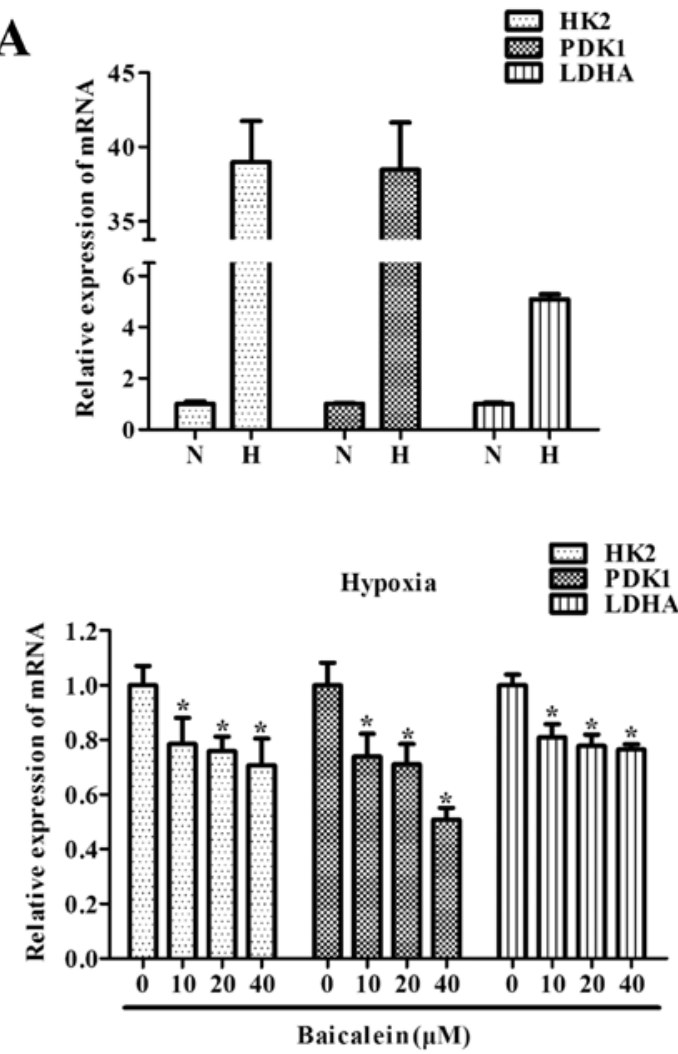

B HK2 PDK1

LDHA

$\beta$-actin

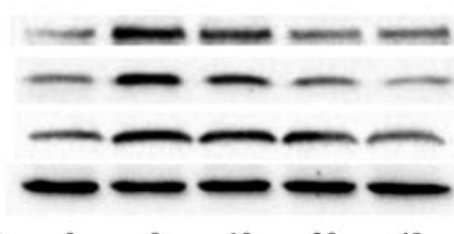

Baicalein $(\mu \mathrm{M})$

Normoxia

Hypoxia

Figure 5. Baicalein inhibits the expression of glycolysis-related enzymes in AGS cells under hypoxia. The AGS cells were treated with the indicated concentrations of baicalein under normoxia $(\mathrm{N})$ or hypoxia $(\mathrm{H})$. (A) The mRNA levels of HK2, LDHA and PDK1 were determined by qPCR. $\beta$-actin was used as the internal control. Data are represented as means \pm SD of three independent experiments. ${ }^{*} \mathrm{P}<0.05$ compared with the normoxic group; ${ }^{*} \mathrm{P}<0.05$ compared with the hypoxic group treated without baicalein. (B) The protein levels of HK2, LDH and PDK1 were determined by western blotting. $\beta$-actin was used as the internal control. Data are representative of three independent experiments. HK2, hexokinase-2; LDHA, lactate dehydrogenase A; PDK1, pyruvate dehydrogenase kinase-1.

AGS cells under a hypoxic condition. As predicted, baicalein suppressed Akt phosphorylation under hypoxia (Fig. 6). In addition, we also found that baicalein facilitated the protein expression of PTEN under hypoxia, which is an upstream negative regulator of the Akt pathway.

As HIF- $1 \alpha$ is one of the target genes mediated by the PTEN/Akt pathway, regulating PTEN/Akt signaling may be the mechanism whereby baicalein inhibits hypoxic induction of HIF-1 $\alpha$ protein expression.

\section{Discussion}

Hypoxia exists in most solid tumors due to an inadequate and poorly formed vasculature. Most conventional chemo-

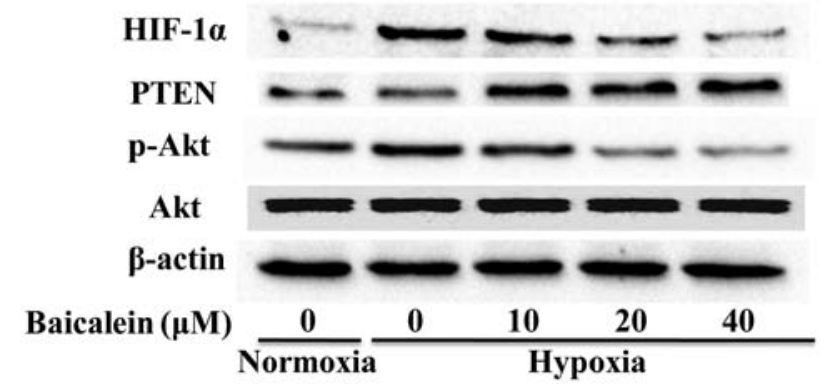

Figure 6. Baicalein inhibits hypoxia-dependent HIF-1 $\alpha$ expression via regulation of the PTEN/Akt pathway in AGS cells. AGS cells were treated with the indicated concentrations of baicalein under normoxia or hypoxia. The protein levels of HIF-1 $\alpha$, PTEN, pAkt and Akt were determined by western blotting. $\beta$-actin was used as the internal control. Data are representative of three independent experiments. HIF-1 $\alpha$, hypoxia-inducible factor-1 $\alpha$.

therapeutic drugs exhibit less effectiveness under hypoxic conditions in cancer treatment (5). Therefore, there is urgent need to seek novel and better sensitizers to overcome hypoxicmediated chemotherapeutic resistance. In the present study, we demonstrated for the first time the potential applicability of baicalein as a novel therapeutic sensitizer to improve the efficacy of 5-FU for the treatment of GC in vitro. We found that baicalein reversed 5-FU resistance induced by hypoxia in AGS cells. Specifically, the doses of baicalein we used were non-cytotoxic or weakly cytotoxic to AGS cells under a hypoxic condition.

Although hypoxia is toxic to cells, malignant cells develop adaptive mechanisms to evade death cause by hypoxia and to resist the assault of chemotherapy. Increased glycolysis is one of the hypoxia-induced phenotypes linked to chemotherapeutic resistance and hypoxia. Glycolysis is the main metabolic pathway for hypoxic cancer cells to generate ATP. Most known proteins involved in the drug resistance phenotype, such as drug efflux pumps, function by relying on ATP. ATP depletion induced by glycolytic inhibition can cause a severe energy crisis, leading to cancer cells becoming more susceptible to chemotherapeutic drugs (24). Moreover, mitochondrial dysfunctions and free radical generation have also been implicated in the apoptosis or necrotic process caused by chemotherapeutic drugs (25-27). Glycolytic metabolism maintains mitochondrial potential in an ATP-dependent manner and scavenges mitochondrial superoxide in an pyruvate-dependent manner, leading to drug failure (28). A previous study showed that dichloroacetate (DCA), a glycolysis inhibitor, was effective in reducing cellular resistance to 5-FU under a hypoxic condition (29). Considering the important role of glycolysis in hypoxia-induced drug resistance, we hypothesized that inhibition of glycolysis may contribute to the reversal effect of baicalein under hypoxia. For verification, we assessed the effect of baicalein on glycolysis and glycolysis-related enzymes in GC cells under a hypoxic condition. As predicted, baicalein enhanced glucose uptake and lactate production under hypoxia gradually back to levels noted under normoxia. This process may be at least partially due to downregulation of HK2, LDHA and PDK1 by baicalein treatment. $\mathrm{HK}$ is the enzyme that controls the first and rate-limiting step of glycolysis, which is the irreversible phosphorylation of glucose to glucose-6-phosphate 
with consumption of ATP. HK2 is the major isoform that is predominantly expressed in a wide range of malignant tumors and is closely correlated with enhanced glycolysis and survival of cancer cells $(30,31)$. LDH is the last glycolytic enzyme that catalyzes the reduction of pyruvate to lactate, the end-product of glycolysis. LDHA is the isoform that possesses the highest efficiency to convert pyruvate to lactate under hypoxic conditions. Previous research demonstrated that inhibition of LDHA expression decreased the proliferation of tumors under hypoxic conditions and stimulated mitochondrial respiration $(32,33)$. Moreover, increased expression of LDHA plays an important role in resistance of human breast cancer cells to taxol and inhibition of LDHA re-sensitizes taxol-resistant cells to taxol (34). PDK1 is a Ser/Thr kinase that inactivates mitochondrial pyruvate dehydrogenase (PDH) by phosphorylation. As PDH is a gatekeeper enzyme that converts pyruvate to acetyl-CoA, production of PDK1 represses mitochondrial function and causes a shift in energy metabolism from oxidative phosphorylation to glycolysis. This process has been demonstrated to prevent the generation of reactive oxygen species, allowing cancer cell survival in hypoxic conditions $(35,36)$. Inhibition of PDK1 has also been found to attenuate hypoxia-induced resistance to 5-FU in GC through regulation of glycolysis (29).

We further focused on HIF-1 since its functions were the most well defined in relation to modifying glycolysis triggered by hypoxia. The molecular mechanisms whereby HIF-1 modulates tumor glycolytic flux were involved in the upregulation of several key glycolytic enzymes including HK2, LDHA and PDK1 $(37,38)$. In the present study, we found that the protein level of HIF-1 $\alpha$ (active subunit of HIF-1) was downregulated by baicalein under a hypoxic condition. A similar finding was obtained by Krishnamoorthy et al in prostate cancer cells (39). Additionally, we investigated the mechanism implicated in the inhibition of HIF-1 $\alpha$ by baicalein. The aberrant activation of the Akt signaling pathway often occurs in hypoxic cancer cells and contributes to the development of malignant phenotypes. Substantial evidence has revealed a mechanism of HIF-1 $\alpha$ regulation by Akt signaling in the process of hypoxia-induced chemoresistance (12-15). PTEN, an upstream negative regulator of Akt, has been suggested as an inhibitor of HIF-1a via inactivation of the Akt pathway $(17,40,41)$. We found that baicalein suppressed hypoxia-induced Akt phosphorylation through promotion of PTEN accumulation in AGS cells. Taken together, baicalein may inhibit hypoxia-dependent HIF-1 $\alpha$ expression via the PTEN/Akt pathway.

In conclusion, we demonstrated that baicalein reversed hypoxia-induced 5-FU resistance through suppression of glycolysis via regulation of the PTEN/Akt/HIF- $\alpha$ signaling pathway. It was also suggested that baicalein may be a potential therapeutic sensitizer which could be applied to increase 5 -FU efficacy in the treatment of GC.

\section{Acknowledgements}

This study was sponsored by the Key Clinical Specialty Discipline Construction Program of Fujian, China, the Medical Elite Cultivation Program of Fujian, China (2013-ZQN-ZD-14), and the Special Funds of the Finance Department of Fujian Province (2012B013).

\section{References}

1. Jemal A, Bray F, Center MM, Ferlay J, Ward E and Forman D: Global cancer statistics. CA Cancer J Clin 61: 69-90, 2011.

2. Hartgrink HH, Jansen EP, van Grieken NC and van de Velde CJ: Gastric cancer. Lancet 374: 477-490, 2009.

3. Zhang YW, Zhang YL, Pan H, et al: Chemotherapy for patients with gastric cancer after complete resection: a network metaanalysis. World J Gastroenterol 20: 584-592, 2014.

4. Longley DB, Harkin DP and Johnston PG: 5-Fluorouracil: mechanisms of action and clinical strategies. Nat Rev Cancer 3: 330-338, 2003.

5. Cosse JP and Michiels C: Tumour hypoxia affects the responsiveness of cancer cells to chemotherapy and promotes cancer progression. Anticancer Agents Med Chem 8: 790-797, 2008.

6. Nakamura J, Kitajima Y, Kai K, et al: HIF-1 $\alpha$ is an unfavorable determinant of relapse in gastric cancer patients who underwent curative surgery followed by adjuvant 5 -FU chemotherapy. Int J Cancer 127: 1158-1171, 2010.

7. Liu L, Ning X, Sun L, et al: Hypoxia-inducible factor-1 $\alpha$ contributes to hypoxia-induced chemoresistance in gastric cancer. Cancer Sci 99: 121-128, 2008.

8. Stoeltzing O, McCarty MF, Wey JS, et al: Role of hypoxiainducible factor $1 \alpha$ in gastric cancer cell growth, angiogenesis, and vessel maturation. J Natl Cancer Inst 96: 946-956, 2004.

9. Maxwell PH, Dachs GU, Gleadle JM, et al: Hypoxia-inducible factor-1 modulates gene expression in solid tumors and influences both angiogenesis and tumor growth. Proc Natl Acad Sci USA 94: 8104-8109, 1997.

10. Denko NC: Hypoxia, HIF1 and glucose metabolism in the solid tumour. Nat Rev Cancer 8: 705-713, 2008.

11. Bhattacharya B, Low SH, Soh C, et al: Increased drug resistance is associated with reduced glucose levels and an enhanced glycolysis phenotype. Br J Pharmacol 171: 3255-3267, 2014.

12. Lau CK, Yang ZF, Ho DW, et al: An Akt/hypoxia-inducible factor-1 $\alpha /$ platelet-derived growth factor-BB autocrine loop mediates hypoxia-induced chemoresistance in liver cancer cells and tumorigenic hepatic progenitor cells. Clin Cancer Res 15: 3462-3471, 2009.

13. Jiao M and Nan KJ: Activation of PI3 kinase/Akt/HIF-1 $\alpha$ pathway contributes to hypoxia-induced epithelial-mesenchymal transition and chemoresistance in hepatocellular carcinoma. Int J Oncol 40: 461-468, 2012.

14. Kilic-Eren M, Boylu T and Tabor V: Targeting PI3K/Akt represses hypoxia inducible factor- $1 \alpha$ activation and sensitizes Rhabdomyosarcoma and Ewing's sarcoma cells for apoptosis. Cancer Cell Int 13: 36, 2013.

15. Zhang J, Guo H, Zhu JS, Yang YC, Chen WX and Chen NW: Inhibition of phosphoinositide 3-kinase/Akt pathway decreases hypoxia inducible factor- $1 \alpha$ expression and increases therapeutic efficacy of paclitaxel in human hypoxic gastric cancer cells. Oncol Lett 7: 1401-1408, 2014.

16. Koshikawa N, Hayashi J, Nakagawara A and Takenaga K: Reactive oxygen species-generating mitochondrial DNA mutation up-regulates hypoxia-inducible factor- $1 \alpha$ gene transcription via phosphatidylinositol 3-kinase-Akt/protein kinase C/histone deacetylase pathway. J Biol Chem 284: 33185-33194, 2009.

17. Zundel W, Schindler C, Haas-Kogan D, et al: Loss of PTEN facilitates HIF-1-mediated gene expression. Genes Dev 14: 391-396, 2000.

18. Huang WH, Lee AR, Chien PY and Chou TC: Synthesis of baicalein derivatives as potential anti-aggregatory and antiinflammatory agents. J Pharm Pharmacol 57: 219-225, 2005.

19. Tsang PW, Chau KY and Yang HP: Baicalein exhibits inhibitory effect on the energy-dependent efflux pump activity in non-albicans Candida fungi. J Chemother: Feb 18, 2014 (Epub ahead of print). doi: http://dx.doi.org/10.1179/1973947814Y.0000000177.

20. Luo R, Wang J, Zhao L, et al: Synthesis and biological evaluation of baicalein derivatives as potent antitumor agents. Bioorg Med Chem Lett 24: 1334-1338, 2014.

21. Song L, Yang H, Wang HX, et al: Inhibition of 12/15 lipoxygenase by baicalein reduces myocardial ischemia/reperfusion injury via modulation of multiple signaling pathways. Apoptosis 19: 567-580, 2014.

22. Taniguchi H, Yoshida T, Horinaka M, et al: Baicalein overcomes tumor necrosis factor-related apoptosis-inducing ligand resistance via two different cell-specific pathways in cancer cells but not in normal cells. Cancer Res 68: 8918-8927, 2008. 
23. Chen J, Xie J, Jiang Z, Wang B, Wang Y and Hu X: Shikonin and its analogs inhibit cancer cell glycolysis by targeting tumor pyruvate kinase-M2. Oncogene 30: 4297-4306, 2011.

24. Tavares-Valente D, Baltazar F, Moreira R and Queirós O Cancer cell bioenergetics and $\mathrm{pH}$ regulation influence breast cancer cell resistance to paclitaxel and doxorubicin. J Bioenerg Biomembr 45: 467-475, 2013.

25. Temkin V, Huang Q, Liu H, Osada H and Pope RM: Inhibition of ADP/ATP exchange in receptor-interacting protein-mediated necrosis. Mol Cell Biol 26: 2215-2225, 2006.

26. Goossens V, Grooten J, De Vos K and Fiers W: Direct evidence for tumor necrosis factor-induced mitochondrial reactive oxygen intermediates and their involvement in cytotoxicity. Proc Natl Acad Sci USA 92: 8115-8119, 1995.

27. Ortiz-Lazareno PC, Bravo-Cuellar A, Lerma-Diaz JM, et al: Sensitization of U937 leukemia cells to doxorubicin by the MG132 proteasome inhibitor induces an increase in apoptosis by suppressing NF-kappa B and mitochondrial membrane potential loss. Cancer Cell Int 14: 13, 2014.

28. Huang CY, Kuo WT, Huang YC, Lee TC and Yu LC: Resistance to hypoxia-induced necroptosis is conferred by glycolytic pyruvate scavenging of mitochondrial superoxide in colorectal cancer cells. Cell Death Dis 4: e622, 2013.

29. Xuan Y, Hur H, Ham IH, et al: Dichloroacetate attenuates hypoxia-induced resistance to 5-fluorouracil in gastric cancer through the regulation of glucose metabolism. Exp Cell Res 321: 219-230, 2014.

30. Wolf A, Agnihotri S, Micallef J, et al: Hexokinase 2 is a key mediator of aerobic glycolysis and promotes tumor growth in human glioblastoma multiforme. J Exp Med 208: 313-326, 2011

31. Pedersen PL, Mathupala S, Rempel A, Geschwind JF and Ko YH: Mitochondrial bound type II hexokinase: a key player in the growth and survival of many cancers and an ideal prospect for therapeutic intervention. Biochim Biophys Acta 1555: 14-20, 2002.

32. Fantin VR, St-Pierre J and Leder P: Attenuation of LDH-A expression uncovers a link between glycolysis, mitochondrial physiology, and tumor maintenance. Cancer Cell 9: 425-434, 2006.
33. Wang ZY, Loo TY, Shen JG, et al: LDH-A silencing suppresses breast cancer tumorigenicity through induction of oxidative stress mediated mitochondrial pathway apoptosis. Breast Cancer Res Treat 131: 791-800, 2012

34. Zhou M, Zhao Y, Ding Y, et al: Warburg effect in chemosensitivity: targeting lactate dehydrogenase-A re-sensitizes taxol-resistant cancer cells to taxol. Mol Cancer 9: 33, 2010.

35. Kim JW, Tchernyshyov I, Semenza GL and Dang CV: HIF-1mediated expression of pyruvate dehydrogenase kinase: a metabolic switch required for cellular adaptation to hypoxia. Cell Metab 3: 177-185, 2006.

36. Papandreou I, Cairns RA, Fontana L, Lim AL and Denko NC: HIF-1 mediates adaptation to hypoxia by actively downregulating mitochondrial oxygen consumption. Cell Metab 3: 187-197, 2006.

37. Marín-Hernández A, Gallardo-Pérez JC, Ralph SJ, RodríguezEnríquez S and Moreno-Sánchez R: HIF-1 $\alpha$ modulates energy metabolism in cancer cells by inducing over-expression of specific glycolytic isoforms. Mini Rev Med Chem 9: 1084-1101, 2009.

38. Velpula KK, Bhasin A, Asuthkar S and Tsung AJ: Combined targeting of PDK1 and EGFR triggers regression of glioblastoma by reversing the Warburg effect. Cancer Res 73: 7277-7289, 2013.

39. Krishnamoorthy S, Jin R, Cai Y, et al: 12-Lipoxygenase and the regulation of hypoxia-inducible factor in prostate cancer cells Exp Cell Res 316: 1706-1715, 2010.

40. Fang J, Ding M, Yang L, Liu LZ and Jiang BH: PI3K/PTEN/AKT signaling regulates prostate tumor angiogenesis. Cell Signal 19: 2487-2497, 2007.

41. Zhong H, Chiles K, Feldser D, et al: Modulation of hypoxiainducible factor $1 \alpha$ expression by the epidermal growth factor/ phosphatidylinositol 3-kinase/PTEN/AKT/FRAP pathway in human prostate cancer cells: implications for tumor angiogenesis and therapeutics. Cancer Res 60: 1541-1545, 2000. 